\title{
Assessing data cybersecurity using ISO/IEC 25012
}

\section{Javier Verdugo ${ }^{1,2}$ (D) $\cdot$ Moisés Rodríguez ${ }^{1,2}$ (D)}

(C) Springer Science+Business Media, LLC, part of Springer Nature 2020

\begin{abstract}
Data is of ever-growing importance and is widely considered to be a company's most valuable asset. Since data is becoming the main driver of business value, data quality and, specifically, data security are of paramount importance to companies. Various regulations related to data cybersecurity have been drawn up, such as the GDPR and the Cybersecurity Act, thus proving the importance placed on data cybersecurity by influential legislative institutions. Several standards related to security have emerged in recent years, most notably those of the ISO/IEC 27000 series. They are, however, focused on management systems and security infrastructure and ignore the security of the data itself. Other standards related to data quality, such as ISO 8000, also fail to address data security in depth. This paper, therefore, proposes a framework for the evaluation of data cybersecurity, consisting of a quality model, an evaluation process, and a tool for the visualization of the assessment results. This evaluation framework has been employed as the basis for a data cybersecurity certification scheme, which complements other certifiable standards related to data and security, such as ISO/IEC 27001 and ISO 8000. This work additionally presents the results of a pilot project in which the data cybersecurity of a commercial product was evaluated. The results of this pilot application allowed us to validate the feasibility of the evaluation framework defined.
\end{abstract}

Keywords Data cybersecurity · Data evaluation · Data certification · ISO/IEC 25012 · GDPR

\section{Introduction}

The increasing emergence of technologies related to open data, big data, business intelligence, etc. has, in recent years, led data to become the most important asset of those companies that have blossomed in the digital era.

This article belongs to the Topical Collection on Quality Management for Information Systems Guest Editors: Mario Piattini, Ignacio García Rodríguez de Guzmán, Ricardo Pérez del Castillo

Javier Verdugo

jverdugo@aqclab.es

1 AQCLab, Camino Moledores, 13005 Ciudad Real, Spain

2 Alarcos Research Group, Institute of Technologies and Information Systems, University of

Castilla-La Mancha, Paseo de la Universidad, 4, 13071 Ciudad Real, Spain 
Other technological advancements that are increasingly present around us every day, such as the Internet of things and artificial intelligence, also rely heavily on the generation and the availability of vast amounts of data. This data, together with the design of groundbreaking algorithms and the increase in computing power, has led to impressive progress in different areas and industries, such as self-driving cars, virtual assistants, or the implementation of automated investment (World Economic Forum 2019).

The growing importance of data as a driver of business value signifies that data security is now an even more relevant concern. One example of this is the political agreement on the Cybersecurity Act reached by the European Parliament, the Council, and the European Commission. Among other things, The Cybersecurity Act creates a framework for European cybersecurity certificates for products, processes, and services that will be valid throughout the EU (European Comission website 2019).

However, although this EU framework for cybersecurity certification is still being implemented, the focus regarding security has, to date, been on infrastructure and management systems, but not on the security of the data itself.

Moreover, the General Data Protection Regulation (GDPR) (European Union law website 2019), which was also drawn up by the European Parliament and the Council of the European Union, addresses the protection of natural persons with regard to the processing of personal data and the free movement of that data, thus highlighting the relevance of the protection and privacy of the personal data of individual citizens in the European Economic Area. The GDPR defines a specific section for the security of personal data, thus establishing the obligation to implement appropriate technical and organizational measures to ensure a level of security appropriate to the risk.

The main objective of this paper is to present the data cybersecurity evaluation framework that has been defined, which consists of a quality model, an evaluation process, and a technological environment. The results of the data cybersecurity evaluations carried out using the framework presented in this work are the basis for data cybersecurity certification, as part of the scheme defined by AENOR (the leading certification body in Spain) for cybersecurity and privacy, which consists of several complementary certifiable standards.

The remainder of the paper is structured as follows: Sect. 2 presents work related to that presented in this paper, and Sect. 3 presents the ISO/IEC 25000 series of standards, which is the basis for the model and process proposed in this work. Section 4 presents the data cybersecurity model defined, while Sect. 5 presents the environment for data cybersecurity evaluation and certification. Section 6 presents a case in which the data cybersecurity evaluation framework was applied, and finally, Sect. 7 presents our conclusions and future work.

\section{Related work}

There are several works (standards, models, best practices, etc.) related to security and data quality, and some considerations regarding the most relevant are shown as follows:

ISO/IEC 27000 (ISO/IEC 27000 2018) is currently the most widespread series of standards for security assessment and certification. Of all the security controls proposed by the standards in this series, several are applicable to data, but the purpose of ISO/IEC 27000 is to evaluate and certify information security management systems (based on the Deming PDCA cycle), and it does not specifically address the evaluation and certification of data security. 
Other standards related to cybersecurity focus on the threats and weaknesses of software systems. One example of this is the Automated Source Code Security Measure (ASCSM) standard (ASCSM 1.0 2016) defined by the OMG, which identifies weaknesses that can be detected in the source code of applications through the use of static analysis. As with other approaches for cybersecurity, it does not specifically address data security.

There are also works related to data quality, although they primarily focus on data management and governance processes, and the exchange of master data. This occurs with, for example, ISO 8000 (ISO/TS 8000-60 2017), the MAMD data maturity model (Carretero et al. 2017), or the work presented in (Rivas et al. 2017). However, none of them provides an explicit approach to security that is focused on data itself. Furthermore, ISO/IEC 25012 (ISO/ IEC 25012 2008) defines a data quality model consisting of several quality characteristics that are applicable to data, and although there is no explicit categorization related to security, some of the quality characteristics are indeed related to data security, as explained in Sect. 4.

With regard to tools related to security, many of them exist, as shown in (Rodríguez et al. 2019). Some of the existing tools include security as part of their broader continuous verification and validation approach, such as SonarQube or CAST Highlight, while others are more specific to security, such as OWASP ZAP, Microfocus Fortify, or Security AppScan. However, security tools focus on analyzing software systems in order to discover their vulnerabilities, but none of them are intended to analyze the security of data in the strict sense.

In summary, the evaluation of data cybersecurity is currently at a very early stage, with no specific global reference standards on the subject. And while there are works related to this matter, such as frameworks for the evaluation of the quality of the software product, the quality of data, the cybersecurity of critical infrastructures and the certification of information security management systems, none of them are specific to data cybersecurity.

\section{ISO/IEC 25000 series of standards}

\subsection{Organization of SQuaRE series of standards}

ISO/IEC 25000 "Software Product Quality Requirements and Evaluation” (ISO/IEC 25000 2014), also known as SQuaRE, is a series of international standards that stems from and revises the old standards related to software product quality, ISO/IEC 9126 (ISO/IEC 9126-1 2001), and ISO/IEC 14598 (ISO/IEC 14598-1 1999). The ISO/IEC 25000 series was devised with the aim of amending the differences and inconsistencies between those two standards and replacing them in order to define a complete framework that establishes, among other things, a quality model, a set of metrics, and a product quality evaluation process.

The standards in the ISO/IEC 25000 family are organized in five divisions, as shown in Fig. 1.

- ISO/IEC 2500n - Quality Management Division. The standards in this division define the common models, terms, and definitions referred to by all the other standards in the SQuaRE series. This division also provides guidance with which to support the functions responsible for the management of software product quality specification and evaluation.

- ISO/IEC 2501n - Quality Model Division. The standards in this division provide detailed models for systems and software product quality and quality in use (ISO/IEC 25010 (ISO/IEC 250102011 )), data quality (ISO/IEC 25012 (ISO/IEC 25012 2008)), and service quality (ISO/ IEC 25011 (ISO/IEC TS 25011 2017)). Guidance on the use of these models is also provided. 


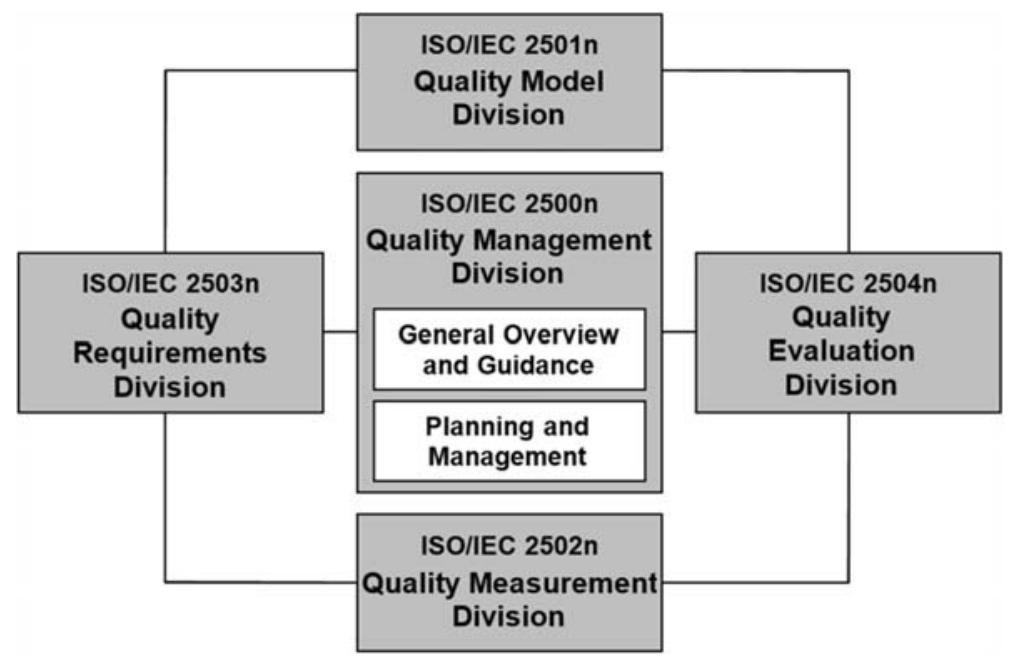

Fig. 1 Organization of the ISO/IEC 25000 series of standards (adapted from (Zubrow 2004))

- ISO/IEC 2502n - Quality Measurement Division. The standards of which this division is formed include a reference model for systems and software product quality measurement (ISO/IEC 25020 (ISO/IEC 25020 2007)), definitions of quality measures for the different models in the ISO/IEC $2501 \mathrm{n}$ division, and practical guidance for their application. In this respect, ISO/IEC 25022 (ISO/IEC 25022 2016) provides measures for quality in use, ISO/IEC 25023 (ISO/IEC 25023 2016) provides those for systems and software product quality, and ISO/IEC 25024 (ISO/ IEC 25024 2015) provides those for data quality. ISO/IEC 25025, which is currently under development, will provide measures for IT service quality.

- ISO/IEC 2503n - Quality Requirements Division. The standards in this division help to specify quality requirements, which can be used in the requirements elicitation process for a system or software product to be developed or as inputs for an evaluation process.

- ISO/IEC 2504n - Quality Evaluation Division. The standards that comprise this division provide a process and requirements for quality evaluation (ISO/IEC 25040 (ISO/IEC 25040 2011)), along with recommendations and guidelines for developers, acquirers, and independent evaluators (ISO/IEC 25041 (ISO/IEC 25041 2012)).

The numeration ISO/IEC 25050 to ISO/IEC 25099 is reserved for the extension to SQuaRe, which is designated to contain system or software product quality standards and/or technical reports that address specific application domains or that can be used to complement the standards in the previous five divisions. For example, ISO/IEC 25051 (ISO/IEC 250512014 ) provides requirements for quality of ready-to-use software product (RUSP) and instructions for their conformity evaluation, and standards in the range ISO/IEC 25060 to ISO/IEC 25066 provide a Common Industry Format (CIF) for usability-related information. 


\subsection{ISO/IEC 25012: data quality model}

ISO/IEC 25012 defines a general data quality model for data that is part of a computer system. Besides the data, computer systems are composed of the hardware and software used for its processing and conservation. Computer systems are, in turn, part of the information systems, which also encompass people and the processes and tasks they carry out as users of the information system.

The quality model in ISO/IEC 25012 defines 15 quality characteristics for data, taking into account that they can apply to all data types (e.g., character strings, texts, dates, numbers, images, sounds, etc.), as long as the data is retained in a computer system and regardless of its physical organization.

The quality characteristics defined in ISO/IEC are categorized according to two points of view:

- Inherent data quality: this refers to quality characteristics that are measured as regards attributes of the data itself.

- System dependent data quality: this refers to quality characteristics that are measured in relation to the capabilities of the computer system retaining the data.

The relationship between the data quality points of view and the domain to which they are related is shown in Fig. 2.

\subsection{ISO/IEC 25024: measurement of data quality}

ISO/IEC 25024 defines the data quality measures that are employed to quantitatively measure data quality in terms of the characteristics defined in ISO/IEC 25012 (shown in Table 1).

For each characteristic defined in ISO/IEC 25012, this standard proposes:

- A basic set of data quality measures

- A basic set of target entities to which the quality measures are applied during the data lifecycle

- An explanation of how to apply the data quality measures

- Guidance for organizations that define their own measures for data quality

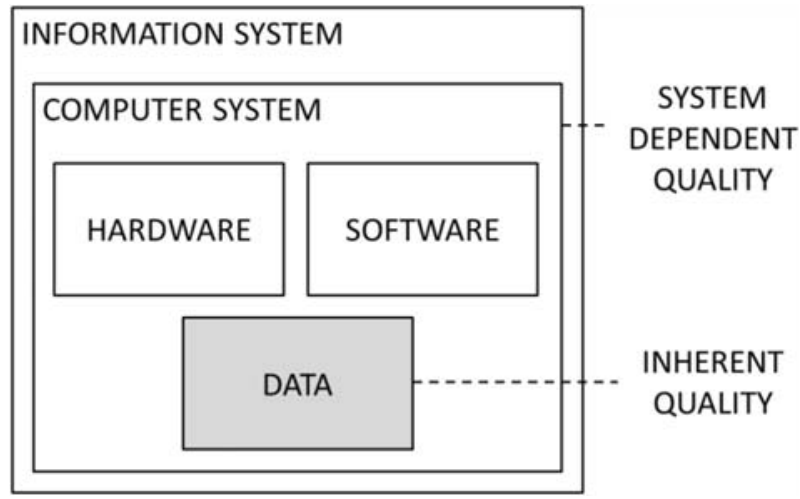

Fig. 2 Relationship between information system, computer system and data 
The classification of the 15 data quality characteristics defined in ISO/IEC 25012 is presented in Table 1 . As the table shows, some characteristics are relevant from both points of view.Table 1 Data quality characteristics defined in ISO/IEC 25012

\begin{tabular}{lll}
\hline Characteristics & Data quality & \\
\cline { 2 - 3 } & Inherent & System dependent \\
\hline Accuracy & $\mathrm{X}$ & \\
Completeness & $\mathrm{X}$ & \\
Consistency & $\mathrm{X}$ & \\
Credibility & $\mathrm{X}$ & $\mathrm{X}$ \\
Currentness & $\mathrm{X}$ & $\mathrm{X}$ \\
Accessibility & $\mathrm{X}$ & $\mathrm{X}$ \\
Compliance & $\mathrm{X}$ & $\mathrm{X}$ \\
Confidentiality & $\mathrm{X}$ & $\mathrm{X}$ \\
Efficiency & $\mathrm{X}$ & $\mathrm{X}$ \\
Precision & $\mathrm{X}$ & $\mathrm{X}$ \\
Traceability & $\mathrm{X}$ & $\mathrm{X}$ \\
Understandability & $\mathrm{X}$ & $\mathrm{X}$ \\
Availability & & \\
Portability & & \\
Recoverability & & \\
\hline
\end{tabular}

\section{Data cybersecurity evaluation model}

The data cybersecurity model presented in this work follows a hierarchical approach. As Fig. 3 shows, the model defines different elements at four levels. The topmost level corresponds to the macrocharacteristic data cybersecurity. On the second level, the model defines quality characteristics, which have been selected from the ISO/IEC 25012 standard (data quality model). The next level defines quality properties, which have been adapted from ISO/IEC 25024 (measurement of data quality). Finally, the bottommost level of the hierarchy corresponds to base measures.

This model has been incorporated into the evaluation framework of AQCLab, an accredited laboratory that carries out evaluations of software product functional suitability (Rodríguez et al. 2016), software product maintainability (Rodríguez and Piattini 2015), and data quality.

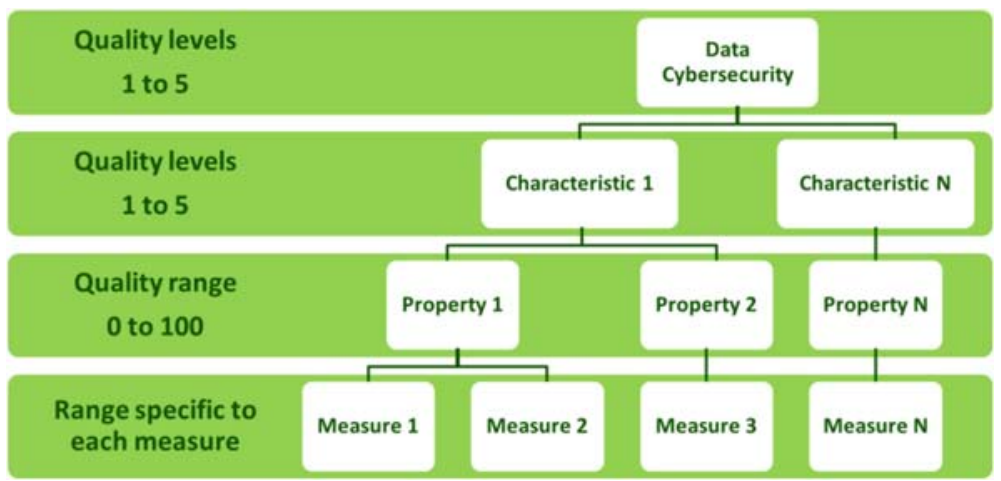

Fig. 3 Hierarchical approach for the data cybersecurity model 


\subsection{Data cybersecurity macrocharacteristic}

This is defined as an aggregate of the different quality characteristics related to data cybersecurity. It takes a value on the scale of 1 to 5, which represents the quality levels for data cybersecurity, ranging from deficient quality to excellent quality.

\subsection{Quality characteristics}

The data cybersecurity model consists of a subset of five characteristics defined in the ISO/IEC 25012 data quality model that were selected for their close relation to security aspects:

- Compliance: degree to which data adhere to standards, conventions, regulations, and similar rules relating to data quality

- Confidentiality: degree to which data is ensured to be accessible and interpretable only by authorized users

- Traceability: degree to which an audit trail is provided regarding access and changes made to the data

- Availability: degree to which data can be retrieved by authorized users and/or applications

- Recoverability: degree to which data maintains and preserves a specified level of operations and quality, even in the event of failure

As with the data cybersecurity macrocharacteristic, each of these characteristics takes a value on the scale of 1 to 5 as a result of its evaluation, which represents its quality level. This value is obtained by applying an aggregation function to the values of its related properties.

\subsection{Quality properties and base measures}

Property values are taken as the basis for the evaluation of the characteristics. The value of each data cybersecurity characteristic is obtained from the values of the various quality properties employed to evaluate them. The quality properties defined in the data cybersecurity model have been extracted from the quality measures proposed in ISO/IEC 25024. The specific properties for each of the characteristics of the data cybersecurity model are shown in Table 2.

The quality properties of the data cybersecurity model take a quality value in the range $[0,100]$. The information on how to obtain the value for each property, in addition to any other descriptive information necessary for their evaluation, has been defined as part of the data cybersecurity model. The model, therefore, characterizes the following information for each property, including information regarding the base measures for its evaluation: related characteristic, property description, point of view, target entity, target attribute, measurement description, calculation formula, scale, value range, and property evaluation function.

Target entities for the different properties of the model are data files (tables), elements of data architecture (contextual schema, data models, data dictionary), computer system (as a whole), and elements of system architecture (database management system, documents, forms, presentation devices). Each of these target entities has quantifiable attributes on which the base measurements for each property are defined.

The quality value for properties is obtained by applying measurement functions to certain base measures. When these base measures are applied at data file level (meaning 
Table 2 Quality properties for the evaluation of data cybersecurity characteristics and their related point of view (I: Inherent quality: S D: System dependent quality)

\begin{tabular}{llll}
\hline Characteristic & Properties & Point of view \\
\cline { 2 - 3 } & & $\mathrm{I}$ & $\mathrm{S}$ D \\
\hline \multirow{2}{*}{ Compliance } & Regulatory compliance of value and/or format & $\mathrm{X}$ & $\mathrm{X}$ \\
& Regulatory compliance due to technology & $\mathrm{X}$ & $\mathrm{X}$ \\
Confidentiality & Encryption usage & $\mathrm{X}$ \\
Traceability & Non-vulnerability & $\mathrm{X}$ \\
& Users access traceability & $\mathrm{X}$ & $\mathrm{X}$ \\
Availability & Data values traceability & $\mathrm{X}$ \\
& Probability of data available & $\mathrm{X}$ \\
Recoverability & Architecture elements availability & $\mathrm{X}$ \\
& Data recoverability ratio & $\mathrm{X}$ \\
\hline
\end{tabular}

that the target entity is, for example, a table in a relational database), they are measured for each file in the data repository, and a categorizing or profiling function is then applied to obtain the property value derived from the measurements for all the files in the data repository. A description of all the properties of the model (indicated in Table 2), and their related base measures, is shown as follows:

\subsection{Compliance: regulatory compliance of value and/or format}

The general information for the property regulatory compliance of value and/or format is shown in Table 3, while details on the base measures involved in the evaluation of this property are provided in Table 4.

Once the base measure has been obtained for each data file, a property evaluation function is applied in order to obtain the value for regulatory compliance of value and/or format in the range [0-100]. In this case, this property value is obtained by applying a profile function.

The first step when applying this evaluation function is that of obtaining the profile for the data repository. This profile represents what percentage of the data files in the repository belongs to each of the profile ranges shown in Table 5. The profiling function takes this profile as input and provides the value for the property as output. The way a profiling function works is defined in more detail in (Rodríguez et al. 2016).

Table 3 General information for the property regulatory compliance of value and/or format

\begin{tabular}{ll}
\hline Property & Regulatory compliance of value and/or format \\
\hline $\begin{array}{l}\text { Characteristic } \\
\text { Description }\end{array}$ & $\begin{array}{l}\text { Compliance } \\
\text { Degree to which data values and/or format comply with specific standards, conventions, or } \\
\text { regulations. The organization is responsible for identifying or establishing with which rules } \\
\text { the data must comply in terms of value and/or format. These rules can be established either } \\
\text { internally, by the organization that owns the data, or by external regulatory bodies }\end{array}$ \\
Point of view & $\begin{array}{l}\text { Inherent } \\
\text { Pand }\end{array}$
\end{tabular}


Table 4 Measurement information for the property regulatory compliance of value and/or format

\begin{tabular}{|c|c|}
\hline Target entity & Data file (table) \\
\hline Target attribute & Data record(row) \\
\hline Measurement description & $\begin{array}{l}\text { Regulatory compliance of value and/or format for a data file is obtained } \\
\text { as the ratio of records of that file whose value for their fields comply } \\
\text { with specific rules, conventions or regulations that have been established }\end{array}$ \\
\hline \multirow[t]{4}{*}{ Calculation formula } & $\mathrm{X}=\mathrm{A} / \mathrm{B}$ \\
\hline & $\mathrm{X}=$ regulatory compliance of value and/or format for data file \\
\hline & $\begin{array}{l}\mathrm{A}=\text { number of records that have values and/or format that conform to } \\
\text { standards, conventions or regulations }\end{array}$ \\
\hline & $\begin{array}{l}\mathrm{B}=\text { number of records that shall conform to standards, conventions or } \\
\text { regulations owing to their value }\end{array}$ \\
\hline Scale & Ratio \\
\hline Value range & {$[0.0-1.0]$} \\
\hline
\end{tabular}

\subsection{Compliance: regulatory compliance due to technology}

Regulatory compliance due to technology is the degree to which data items comply with specific standards, conventions, or regulations relating to technological aspects of the computer system retaining the data. These rules can be established, either internally by the organization that owns the data or by external regulatory bodies.

Its base measure is calculated for each data file in the repository, thus obtaining the ratio of records of that file whose value for their fields comply with specific rules, conventions, or regulations that have been established. The value of the property is obtained by applying a profiling function, taking the base measure for each file as input.

\subsection{Confidentiality: encryption usage}

Encryption usage is the degree to which data values fulfill requirements regarding encryption. These requirements must establish, for each data file in the repository, which specific fields must contain encrypted data values.

The data to be encrypted is not limited to personal or confidential information. Any other information can be encrypted based on the specifications/requirements of a system or compliance with a law or regulation.

Its base measure is obtained for each data file as the ratio of records whose fields contain data conveniently encrypted according to the established requirements. The value for the property is obtained by applying a profiling function to the base measure values for each file.

Table 5 Profile ranges for the evaluation of regulatory compliance of value and/or format

\begin{tabular}{ll}
\hline Range & Description \\
\hline$[0.0-0.6)$ & Low regulatory compliance of value and/or format \\
{$[0.6-0.75)$} & Medium regulatory compliance of value and/or format \\
{$[0.75-0.95)$} & High regulatory compliance of value and/or format \\
{$[0.95-1.0]$} & Very high regulatory compliance of value and/or format \\
\hline
\end{tabular}




\subsection{Confidentiality: non-vulnerability}

Non-vulnerability is the degree to which data items defined as confidential can be accessed by authorized users only. Formal penetration testing attempts have to be carried out for this purpose.

The target entity for its base measure is the data repository as a whole, and its value is obtained as the ratio of failed accesses to the data repository by unauthorized users with respect to attempted accesses. The value for non-vulnerability is obtained by normalizing the value for the base measure in the range $[0,100]$.

\subsection{Traceability: users access traceability}

Users access traceability is defined as the degree to which the information concerning users' access to the data value was traced by means of either information contained in the data repository itself or other system capabilities (e.g., log files).

User access traceability requirements must be defined by the organization, indicating which files (or which specific fields within them) require traceability as regards access to data values.

Its base measure is calculated for each data file in the repository, obtaining the ratio of records for which there is traceability information concerning users who have accessed its value (for the purpose of either reading or modifying it) over the total number of records for which user access traceability is expected. The value for the property is obtained by applying a profiling function to the base measure values for each file.

\subsection{Traceability: data values traceability}

Data values traceability represents the degree to which it is possible to trace the history of a data item value, by means of either information contained in the data repository itself or other system capabilities.

Data values traceability requirements must be defined by the organization beforehand as regards which files (or which specific fields within them) should maintain a history of the data values of its records.

The value for this property is obtained by applying a profiling function to the values for its base measure, which is calculated for each data file. This base measure represents the ratio of records for which there is traceability information concerning the values that the data has taken throughout its lifecycle (value history) with respect to the total number of records for which this traceability should exist.

\subsection{Availability: data availability}

Data availability is the probability of successful requests attempting to use data items for a specific duration.

The specific duration of the measurement or the period during which it is performed may be during normal processing or at other specific times (such as during backup or restore procedures).

The base measure for this property is the ratio of successful attempts to access data elements in a specific duration over the total number of access attempts made during that period. The target entity for this base measure is, therefore, the data repository as a whole. The value for data availability is obtained by normalizing the value for the base measure in the range $[0,100]$. 


\subsection{Availability: architecture elements availability}

Architecture elements availability represents the degree to which architecture elements are available.

The measurement of this property verifies that the different elements of architecture (user interface, business workflow, data access process, communication process, data quality rules, contextual schema, conceptual, logical, physical data models, data dictionary, etc.) are available for the intended users at a given time.

The base measure for this property is the ratio of how many of these architectural elements are available in relation to the total number of architectural elements. The value for architecture elements availability is obtained by normalizing the value for this base measure in the range $[0,100]$.

\subsection{Recoverability: data recoverability ratio}

Data recoverability ratio is the degree to which data stored in a device are successfully and correctly recovered.

When measuring this property, it is necessary to carry out the data recovery process of a system (restoration of backups, commit/syncpoint, rollback, etc.) in order to verify how many data elements in that system are effectively recovered in that process when compared to the total number of data elements that should be recovered.

The base measure is, therefore, the ratio of data elements recovered correctly over the total number of elements that should be recovered, and the value of the property is obtained by normalizing that ratio in the range $[0,100]$.

\subsection{Recoverability: periodical backup}

Periodical backup evaluates the extent to which periodic data backups are carried out as required by the requirements. These requirements must have been established by the organization, specifying which files should be backed up and how often.

In order to measure this property, it is necessary to examine the existing backups so as to verify how many data elements in the system are contained in those backups versus the total number of data elements that should be backed up.

The effectiveness of the periodicity used in the backups is verified by comparing the contents of a backup with the contents of the evaluated repository at the closest time possible prior to the next backup. This is the point at which there may be greater desynchronization between the repository and the backup, and if there is a problem with the repository, it could have a greater impact on information loss.

The value for this property is obtained by applying a profiling function to the values for its base measure, which is calculated for each data file. This base measure represents the ratio of data elements that have been successfully backed up for each data file specified by the requirements.

\subsection{Recoverability: architecture recoverability}

Architecture recoverability represents the degree to which architecture elements are recoverable. 
When measuring this property, it is necessary to examine the existing backups for elements of the architecture and to carry out their restoration in order to verify how many of those elements of the architecture are successfully recovered when compared to the total number of elements that should be backed up.

The base measure for this property is the ratio of successfully recovered elements of the architecture over the total number of existing elements. This value is then normalized in the range $[0,100]$ in order to obtain the value for the architecture recoverability property.

\section{Data cybersecurity environment}

A data cybersecurity evaluation should be carried out in a methodological, and technological environment is needed. The evaluation model is the main part of the evaluation environment, but in order to be practical, it must be supported by two elements: a set of processes and activities that establish the steps that have to be carried out and guide the interaction with the customers and a set of tools with which to perform the measurements and evaluations and visualize the results.

The purpose of the data cybersecurity environment is, therefore, to establish a basis that will provide companies and public organisms with data cybersecurity evaluation and certification services.

The methodological part of the environment consists of defining the following:

- The set of processes required to carry out data cybersecurity evaluations, the specific activities to be carried out, and the artifacts that are handled during the evaluation process (inputs to the process, outputs or deliverables, etc.).

- The set of processes required to carry out data cybersecurity certification, based on a previous evaluation. This process is analogous to that described in (Rodríguez and Piattini 2015) for the certification of software product quality, but the object being certified is a data repository rather than a software product.

\subsection{Evaluation process}

The evaluation process establishes the activities, inputs, outputs, resources, and constraints required to carry out a data cybersecurity evaluation, in this case, from the point of view of an independent evaluator. The evaluation process defined in this work has been adapted from the process in the ISO/IEC 25040 standard.

The activities and tasks of the data cybersecurity evaluation process (see Fig. 4) are defined as follows. It should be noted that the activities are not carried out strictly one after another. Iterations on specific activities or between activities may and usually occur.

Activity 1: Establish the evaluation requirements The following takes place in this activity: the purpose of the data cybersecurity evaluation is documented; the stakeholders' specific requirements are identified and incorporated into the full set of data cybersecurity requirements for the evaluation, together with the base requirements stated by the evaluation model; and all the data and computer system parts to be included in the evaluation are identified and documented. 


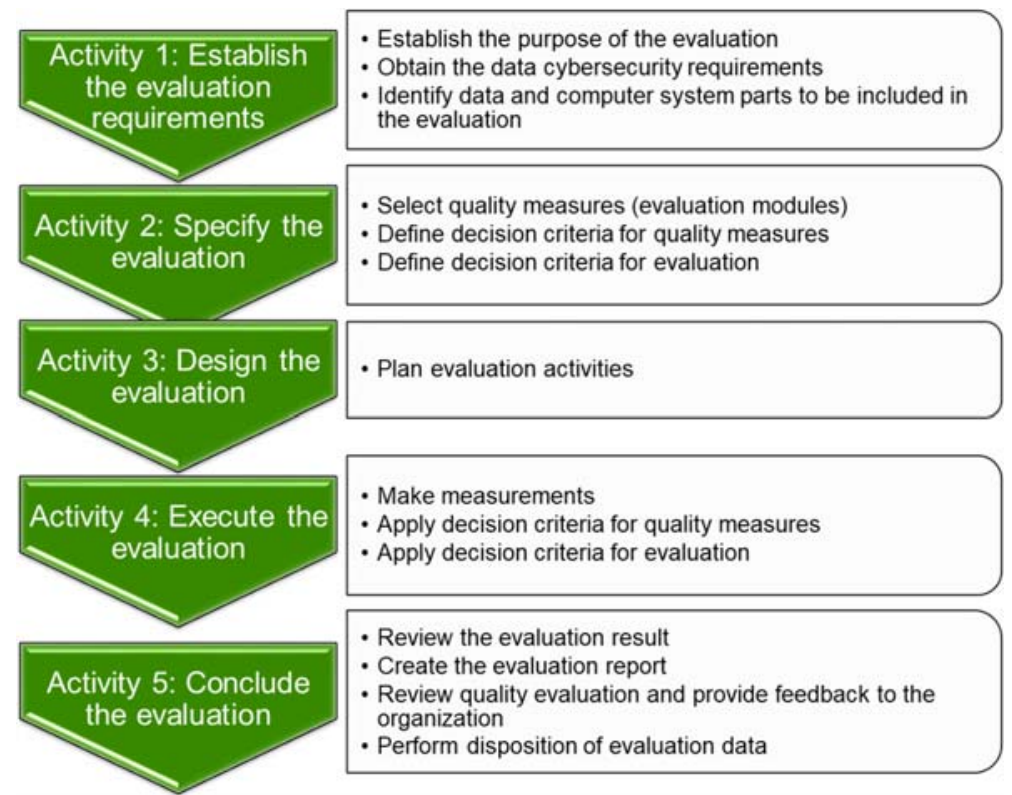

Fig. 4 Evaluation process for data cybersecurity evaluation (adapted from (ISO/IEC 25040 2011))

Activity 2: Specify the evaluation This activity requires the selection of quality measures (evaluation modules), thus making it possible to define the decision criteria (numerical thresholds and targets) for the individual measures and also the decision criteria in order to obtain the value of quality properties and characteristics from those individual measures. However, since the measures of the model are pre-established, in this activity, the evaluator identifies the mapping between the measures, the data and computer system parts, and the data cybersecurity requirements.

Activity 3: Design the evaluation The evaluation activities are scheduled, taking into account the availability of resources and the data and computer system parts to be evaluated.

Activity 4: Execute the evaluation The data cybersecurity measures are applied to the data and computer system parts. The decision criteria for the measures are then applied to the measured values, obtaining values for the quality properties. Finally, the set of decision criteria for the characteristics are applied by employing the property values as an input, which allows the evaluation results to be obtained.

Activity 5: Conclude the evaluation The evaluator generates and provides the client with the evaluation report, detailing the results and including descriptive information concerning the evaluation (requirements, evaluation modules, plan, personnel, etc.). The evaluator and the client may then carry out a joint review of the evaluation results. The evaluator also carries out an internal evaluation quality review in order to improve the evaluation process and evaluation techniques (evaluation modules). Finally, the data and any other items that may have been provided by the client are eliminated from the systems used in the evaluation in a secure manner. 


\subsection{Technological environment}

A technological environment is necessary in order to allow the evaluation of data cybersecurity to be carried out in a practical, efficient, and accurate manner. This technological environment consists of tools that automate the acquisition, calculation, and presentation of the values obtained for the characteristics, properties, and measures defined in the evaluation model.

The measurement tools used in the evaluation to obtain the values for base measures greatly depend on the technologies of the computer system of the data product evaluated. For example, in the case of relational databases, a query tool can be used to communicate with the DBMS and obtain the information required for certain measures of the model.

The evaluation tool for the environment is responsible for applying the decision criteria of the evaluation model. It does this by taking the measurement of the base measures defined in the model as input, once they have been obtained from the target entities. The evaluation tool takes the base measure values specified in a JSON file and processes them to obtain the values for quality properties. This processing consists of applying evaluation functions that represent the decision criteria defined in the model as thresholds and profiles. The values for the quality properties are, in turn, processed to obtain the values for the characteristics of the model. The resulting values for the properties and characteristics after the evaluation are stored in a database.

A visualization tool has been developed in order to consult and represent the results of data cybersecurity evaluations in a clear and concise manner. This tool is intended to be used by both evaluators and clients. The permission system of the tool allows it to control which functionalities and information of which evaluations the user can access based on his/her role. When the user selects an evaluation (from among those available), its information is displayed (see Fig. 5): name of the data product, version, date of the evaluation, value for characteristics, and properties in tabular manner, along with graphics representing those values (radar chart for characteristics, bar chart for properties).

The visualization tool provides more functionalities, such as generating downloadable reports for evaluations, showing trends in the values of cybersecurity evaluations, and managing companies, data products and users, etc.

\section{Example of application}

As a pilot project, the evaluation framework presented in this work has been applied to an existing commercial product with the aim of validating the initial framework defined and verifying the feasibility of conducting data cybersecurity evaluations with this framework. This was done with the intention of allowing us to identify possible drawbacks regarding the measures and their applicability to real-life information systems and to make other general improvements to the framework.

The product selected for this pilot project was a business dashboard management tool provided to its users in the form of a SaaS model. This tool uses the balanced scorecard (BSC) approach, thus allowing the users to define, monitor, and control their own KPIs through the use of a visual dashboard.

The data managed by this product, therefore, correspond to:

- Information regarding the users' accounts (name, email, username, password, company, country, etc.) 


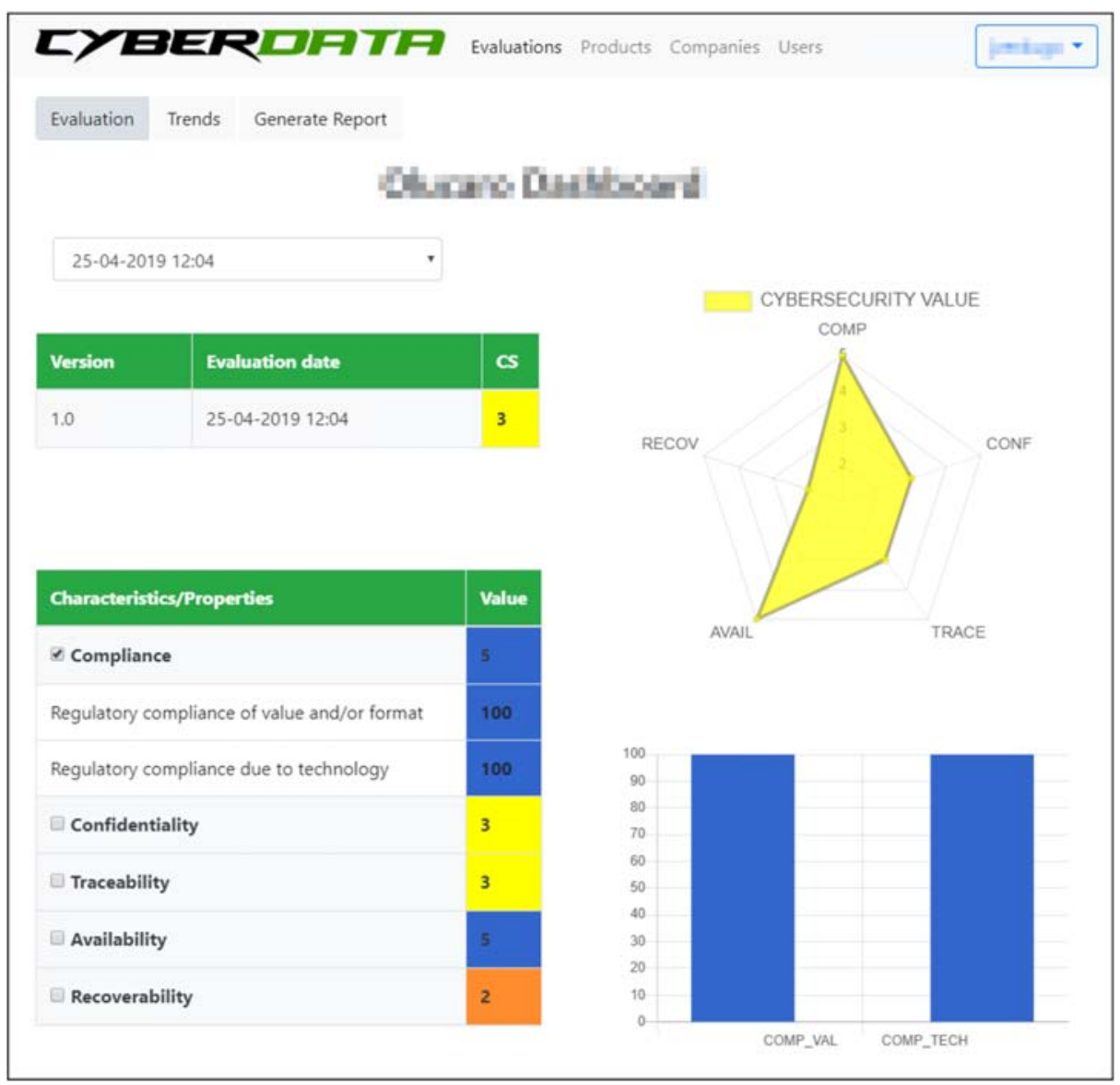

Fig. 5 Snapshot of the visualization tool for data cybersecurity evaluations

- Permissions, managed through the use of ACLs

- Information concerning the specific KPIs defined by the users

- Information regarding the perspectives and objectives defined by the users in order to group and classify the KPIs

- Measurements for the KPIs

- Different aspects of system configuration and user preferences

- $\quad$ System logs

The evaluation was conducted by following the process and using the technological environment defined in the previous section and by applying the model defined in Sect. 4.

Two iterations of the evaluation process were carried out: the first to obtain the data cybersecurity for the product as it was originally implemented and used and the second after making improvements to the system and the data, taking into account those aspects that yielded the worst values for the data cybersecurity properties and characteristics. 


\subsection{First iteration of the evaluation}

The first iteration of the evaluation process had two objectives: to verify that the framework can be effectively used in the evaluation of a real product and to provide a "snapshot" of the data cybersecurity of the product evaluated as a result of this. This was particularly relevant when considering that the specificities of the evaluation framework were not known by the developers during its implementation and use up to that point.

As a result of the first activity, establish the evaluation requirements, the cybersecurity model was presented to the client, and the characteristics, the properties and the generic requirements related to their measures were explained. The client identified the data and elements of the computer system in the scope of the evaluation:

- The database of the application, with all the tables being part of the target for the evaluation

- The application itself, hosted in a server managed by the client

- The DBMS of the application

- The latest backup copies made

- The results of penetration testing carried out

- Documentation of the application and computer system

Specific requirements for certain characteristics were also identified by the client, such as rules regarding value or format for some data fields, fields that must contain encrypted data with a specific algorithm, the frequency of the backups, etc.

As a result of the second activity, specify the evaluation, since the measures for the evaluation had already been defined, the mapping between the measures, the data, and computer system parts and the specific requirements was carried out.

The third activity, design the evaluation, resulted in the evaluation plan, with the allocation of resources to activities.

The fourth activity, execute the evaluation, was then carried out. The base measurements were performed on the target entities according to their specification in the model and the specific requirements identified by the client for certain quality properties. The results of the measurement were processed with the evaluation tool in order to obtain the values for properties and characteristics. The results obtained for the characteristics are shown in Table 6. A value of 2 was obtained for the data cybersecurity macrocharacteristic, which was derived from the values of the characteristics.

In order to finish the first iteration, as part of the conclude the evaluation activity, the evaluation report was generated with the visualization tool and issued to the client. The results of the evaluation were then reviewed with the client.

Table 6 Quality values for the data cybersecurity characteristics obtained in the first iteration of the evaluation

\begin{tabular}{ll}
\hline Characteristic & Value \\
\hline Compliance & 4 \\
Confidentiality & 2 \\
Traceability & 2 \\
Availability & 5 \\
Recoverability & 2 \\
\hline
\end{tabular}


The review of the results of the evaluation with the client made it possible to identify the main problems indicated by the values of the characteristics and properties obtained.

- The value for compliance was affected by some requirements as regards the value and the format of the data not being fully met. For example, the requirement establishing the rule that usernames and email addresses for user accounts cannot be duplicated was violated in some cases.

- The value for confidentiality was severely impacted by the fact that penetration tests had not been performed, which resulted in the quality property non-vulnerability obtaining a value of 0 .

- Traceability owed its value to the value of the property data values traceability. Some of the tables whose objective was to register the log of changes to perspectives and objectives were not actually saving that information and were consequently not meeting the requirements established by the client regarding the need to maintain a history of changes for these entities.

- Recoverability had a low value because of the properties periodical backup and architecture recoverability. In the first case, the frequency of the backups resulted in the information in several of the tables backed up (e.g., those storing users and links among objectives) being significantly desynchronized with respect to the information in the production environment at the moment right before carrying out the following backup process. In the second case, the elements of the architecture identified for the product were not being backed up.

A further analysis of these problems led to the identification of improvements that should be made to the product and its data. Some examples of the improvements identified are:

- Perform data cleansing: deleting duplicate accounts not meeting compliance requirements after consulting the owners of said accounts as to which one is their main account.

- Perform penetration testing. Taking into account that the range of depth for this type of testing is quite wide, an adequate scope is required for these tests.

- Correctly configure the history log for the required entities. As a drawback, this would help from the moment it is implemented, but information prior to this point would still be unavailable as it was not recorded in the first place.

- Increase the frequency of backups. Reducing the time between backups would mean a higher synchronization between the data contained in the backup copies and the data in the production environment, thus reducing the risk of data losses.

- Incorporate architecture elements as part of the backup system.

Since the clients were willing to improve the data cybersecurity evaluation results, they committed to work on some of the improvement actions identified. With regard to other improvements, such as implementing penetration testing, the client decided not to tackle them in the short term, since a lack of experience in this matter would not allow them to carry out the actions themselves, and the costs of externalizing the service were not viable at that moment.

\subsection{Second iteration of the evaluation}

A second iteration of the evaluation was carried out after some of the improvement actions identified after the first iteration were implemented, in order to assess how those improvements might have an impact in the evaluation results. 
The process carried out in the second iteration was similar to that for the first iteration, although not as complex for some of the activities, since some tasks were unnecessary.

This meant that the first activity, establish the evaluation requirements, in this second iteration only required identifying the elements in the scope of the evaluation that changed with respect to the first iteration. A new clone of the database was, therefore, obtained, along with the newest version of the backup copies.

For the second activity, specify the evaluation, the mapping between the measures, the data, and computer system parts and specific requirements was reviewed in order to verify that no changes were necessary.

In the third activity, design the evaluation, the evaluation plan for the second iteration was prepared.

The fourth activity, execute the evaluation, was then performed, obtaining the values for the measures in the new version of the inputs for the evaluation. The values for the properties, characteristics, and overall data cybersecurity were then obtained automatically with the evaluation tool, resulting in a value of 3 for the data cybersecurity macrocharacteristic, and the values that are shown in Table 7 for the characteristics.

These results show that the improvements made by the client were indeed reflected in the higher-quality values obtained for some characteristics and the overall data cybersecurity.

Finally, the activity conclude the evaluation was conducted, during which a new evaluation report was generated and the results were reviewed with the client. The elements provided for the evaluation by the client were then eliminated from the laboratory systems, and a review of the results of the pilot project was conducted.

\subsection{Analysis of the results of the pilot project}

This pilot project, along with the two iterations of the evaluation process performed, allowed us to conclude that the framework defined on the basis of several standards from the ISO/IEC 25000 series can be applied in order to evaluate different aspects of data cybersecurity.

In this case, given the characteristics of the product evaluated, the data cybersecurity evaluation proved to be feasible for web applications whose data is managed centrally in a relational database.

The quality values obtained in both iterations carried out also show that the evaluation model is sensitive to changes in aspects related to data cybersecurity. The second evaluation, therefore, yielded better quality values after several improvements were made to the data in the application under evaluation and the system that retains these data.

Table 7 Quality values for the data cybersecurity characteristics obtained in the second iteration of the evaluation

\begin{tabular}{ll}
\hline Characteristic & Value \\
\hline Compliance & 5 \\
Confidentiality & 2 \\
Traceability & 3 \\
Availability & 5 \\
Recoverability & 4 \\
\hline
\end{tabular}




\section{Conclusions and future work}

This work presents a first step in a domain that is becoming more and more important but has not yet been explored in depth. Although there are many approaches for data quality and cybersecurity, such as standards, models, best practices, etc., none of them actually take into account both at the same time. Data quality approaches do not usually address security aspects comprehensively, and cybersecurity approaches usually focus more on systems infrastructure, software, and networks than on the data.

This work has presented an approach for data cybersecurity that is applicable to the industry and is based on international standards. The pilot project conducted has shown that the proposed framework can be used to evaluate and certify the cybersecurity of the data that companies manage and work with as part of their business mission.

In the future, we intend to carry out more evaluations with this framework in order to obtain more practical knowledge about the feasibility of this type of evaluation, the drawbacks regarding the measures selected, and how to apply them in different types of real-life information systems and, in general, make improvements to the data cybersecurity evaluation framework proposed, thus enabling us to confirm that it addresses the needs of the market.

Since AQCLab is already accredited to ISO/IEC 17025 as a laboratory for software product and data quality evaluation tests in conformance with ISO/IEC 25000, one goal for the future is to incorporate data cybersecurity evaluations into the portfolio of the accredited tests performed by the laboratory.

Funding information This research is part of the DQIoT project (INNO-20171086), funded by CDTI;ECD project (PT3Q-16-08504), funded by the "Torres Quevedo" Program of the Spanish Ministry of Economy, Industry and Competitiveness; the CYBERDATA project (REF: (ISO/IEC 14598-1 1999)/17/IN/013) funded by Consejería de Economía, Empresas y Empleo JCCM and FEDER (Fondo Europeo de Desarrollo Regional); the ECLIPSE project (Ministerio de Ciencia, Innovación y Universidades, and Fondo Europeo de Desarrollo Regional FEDER, RTI2018-094283-B-C31); and the TESTIMO project (Consejería de Educación, Cultura y Deportes de la Junta de Comunidades de Castilla La Mancha, and the Fondo Europeo de Desarrollo Regional FEDER, SBPLY/17/180501/000503).

\section{References}

ASCSM 1.0: Automated Source Code CISQ Security Measure. Object Management Group (2016).

Carretero, A. G., Gualo, F., Caballero, I., \& Piattini, M. (2017). MAMD 2.0: Environment for data quality processes implantation based on ISO 8000-6X and ISO/IEC 33000. Computer Standards and Interfaces, 54, $139-151$.

European Comission website, https://ec.europa.eu/commission/news/cybersecurity-act-2018-dec-11_en, last accessed 2019/10/09.

European Union law website, https://eur-lex.europa.eu/eli/reg/2016/679/oj, last accessed 2019/10/09.

ISO/IEC 14598-1: Information technology - Software product evaluation - Part 1: General overview. International Organization for Standardization / ISO/IEC JTC 1/SC 7 Software and systems engineering (1999).

ISO/IEC 25000: Systems and software engineering - Systems and software Quality Requirements and Evaluation (SQuaRE) - Guide to SQuaRE. International Organization for Standardization / ISO/IEC JTC 1/SC 7 Software and systems engineering (2014).

ISO/IEC 25010: Software Engineering - Software product Quality Requirements and Evaluation (SQuaRE) System and software quality models. International Organization for Standardization / ISO/IEC JTC 1/SC 7 Software and systems engineering (2011). 
ISO/IEC 25012: Software Engineering - Software product Quality Requirements and Evaluation (SQuaRE) Data Quality Model. International Organization for Standardization / ISO/IEC JTC 1/SC 7 Software and systems engineering (2008).

ISO/IEC 25020: Software Engineering - Software product Quality Requirements and Evaluation (SQuaRE) Measurement reference model and guide. International Organization for Standardization / ISO/IEC JTC 1/ SC 7 Software and systems engineering (2007).

ISO/IEC 25022: Software Engineering - Software product Quality Requirements and Evaluation (SQuaRE) Measurement of quality in use. International Organization for Standardization / ISO/IEC JTC 1/SC 7 Software and systems engineering (2016).

ISO/IEC 25023: Software Engineering - Software product Quality Requirements and Evaluation (SQuaRE) Measurement of system and software product quality. International Organization for Standardization / ISO/ IEC JTC 1/SC 7 Software and systems engineering (2016).

ISO/IEC 25024: Software Engineering - Software product Quality Requirements and Evaluation (SQuaRE) Measurement of data quality. International Organization for Standardization / ISO/IEC JTC 1/SC 7 Software and systems engineering (2015).

ISO/IEC 25040: Software Engineering - Software product Quality Requirements and Evaluation (SQuaRE) Evaluation process. International Organization for Standardization / ISO/IEC JTC 1/SC 7 Software and systems engineering (2011).

ISO/IEC 25041: Software Engineering - Software product Quality Requirements and Evaluation (SQuaRE) Evaluation guide for developers, acquirers and independent evaluators. International Organization for Standardization / ISO/IEC JTC 1/SC 7 Software and systems engineering (2012).

ISO/IEC 25051: Software Engineering - Software product Quality Requirements and Evaluation (SQuaRE) Requirements for quality of Ready to Use Software Product (RUSP) and instructions for testing. International Organization for Standardization / ISO/IEC JTC 1/SC 7 Software and systems engineering (2014).

ISO/IEC 27000: Information technology - Security techniques - Information security management systems Overview and vocabulary. International Organization for Standardization / ISO/IEC JTC 1/SC 27 Information Security, cybersecurity and privacy protection (2018).

ISO/IEC 9126-1: Software engineering - Product quality - Part 1: Quality model. International Organization for Standardization / ISO/IEC JTC 1/SC 7 Software and systems engineering (2001).

ISO/IEC TS 25011: Software Engineering - Software product Quality Requirements and Evaluation (SQuaRE) Service quality models. International Organization for Standardization / ISO/IEC JTC 1/SC 7 Software and systems engineering (2017).

ISO/TS 8000-60: Data Quality - Part 60: Data Quality Management: Overview. International Organization for Standardization / TC 184/SC 4 Industrial data (2017).

Rivas, B., Merino, J., Caballero, I., Serrano, M. A., \& Piattini, M. (2017). Towards a service architecture for master data exchange based on ISO 8000 with support to process large datasets. Computer Standards and Interfaces, 54, 94-104.

Rodríguez, M., \& Piattini, M. (2015). Fernandez, C. M.:A hard look at software quality: Pilot program uses ISO/ IEC 25000 family to evaluate, improve and certify software products. Quality Progress, 48, 30-36.

Rodríguez, M., Oviedo, J. R., \& Piattini, M. (2016). Evaluation of Software Product Functional Suitability: A Case Study. Software Quality Professional, 18(3), 18-29.

Rodríguez, M., Piattini, M., \& Ebert, C. (2019). Software verification and validation technologies and tools. IEEE Software, 36(2), 13-24.

World Economic Forum, https:/www.weforum.org/agenda/2016/01/the-fourth-industrial-revolution-what-itmeans-and-how-to-respond/, last accessed 2019/10/09.

Zubrow, D. (2004). Measuring software product quality: The ISO 25000 series and CMMI. SEI.

Publisher's note Springer Nature remains neutral with regard to jurisdictional claims in published maps and institutional affiliations. 


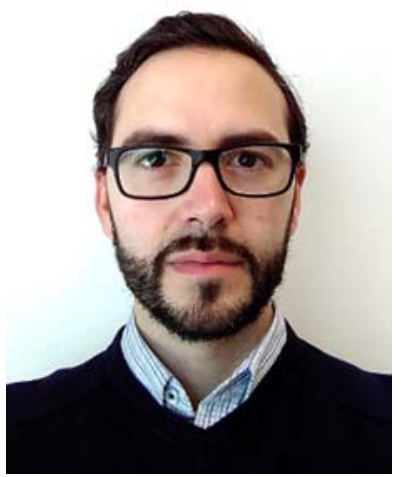

Javier Verdugo has a degree in Computer Science from the University of Castilla-La Mancha. He is Technical director of the accredited laboratory for software and data quality evaluation AQCLab. He is also Chief auditor for AENOR in Software Engineering and R\&D\&I certifications, and since 2017 he has been collaborating as a part time professor Escuela Superior de Informática of the University of Castilla-La Mancha. He is a Certified Information System Auditor for the ISACA, and his research interests are systems security, and software process, software product and data quality evaluation.

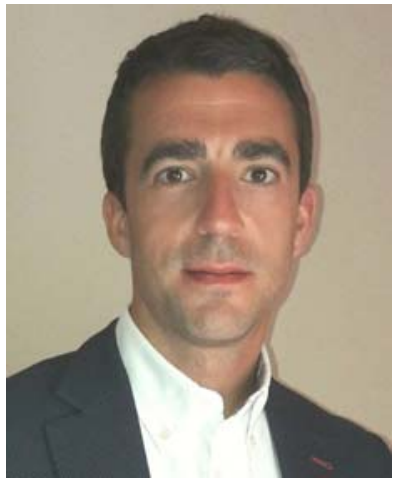

Moisés Rodríguez is a M.Sc. and has a PhD in Computer Science from the University of Castilla-La Mancha. $\mathrm{He}$ is the CEO of the accredited laboratory for software quality assessment AQCLab, is a Certified Information System Auditor for the ISACA (Information System Audit and Control Association) and is a lead Software Engineering Auditor for AENOR. He is a part time Professor at the Escuela Superior de Informática of the University of Castilla-La Mancha. He is part of the ALARCOS research group, and his research interests are: software process, software product and data quality assessment, improvement, and certification. 